\title{
EcoPred: an educational individual based model to explain biological control, a case study within an arable land
}

Jacinto Benhadi-Marín , José Alberto Pereira , José Paulo Sousa \& Sónia A.P. Santos

To cite this article: Jacinto Benhadi-Marín , José Alberto Pereira , José Paulo Sousa \& Sónia A.P. Santos (2020) EcoPred: an educational individual based model to explain biological control, a case study within an arable land, Journal of Biological Education, 54:3, 271-286, DOI: 10.1080/00219266.2019.1569086

To link to this article: https://doi.org/10.1080/00219266.2019.1569086

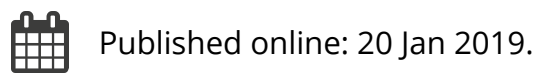

Submit your article to this journal $\pi$

II Article views: 116

Q View related articles ¿

View Crossmark data 


\title{
EcoPred: an educational individual based model to explain biological control, a case study within an arable land
}

\author{
Jacinto Benhadi-Marín (iD) ${ }^{a, b}$, José Alberto Pereira (iD) ${ }^{a}$, José Paulo Sousa $\mathbb{D D}^{\mathrm{b}}$ and Sónia A. \\ P. Santos $\left(\mathbb{D}^{c, d}\right.$
}

aCentro de Investigação de Montanha (CIMO), ESA, Instituto Politécnico de Bragança, Campus de Santa Apolónia, Bragança, Portugal; ${ }^{b}$ Centre for Functional Ecology, Department of Life Sciences, University of Coimbra, Calçada Martim de Freitas, Coimbra, Portugal; 'ClQuiBio, Barreiro School of Technology, Polytechnic Institute of Setúbal, Lavradio, Portugal; dLEAF, Instituto Superior de Agronomia, Lisboa, Portugal

\begin{abstract}
Individual based models (IBMs) are up-to-date tools both in research and educational areas. Here we introduce an IBM built on NetLogo platform that simulates a top-down trophic cascade controlled by the pressure exerted by two model predators (web-building spiders and ground runner spiders) on a model pest (the olive fruit fly) within a hypothetical agricultural landscape (the olive crop). EcoPred is an IBM that aims to be an educational tool that can help teachers to explain concepts related to ecology in a modern, enjoyable and comprehensive way. EcoPred reflects the changes on a fly population within a simulated olive crop according to (1) the mortality rate caused by the predation of two spider species and energy loss, (2) the energy gain by feeding on flowers and (3) the reproduction rate in olive trees. The model was tested with 26 students achieving very good results in terms of acceptance and interest on the learning method. EcoPred can be used for educational purposes with 16 year old students and older to explain ecological concepts such as trophic level, species interactions, limiting factor and biological control in an interactive way simultaneously introducing students to biology oriented programming languages.
\end{abstract}

\section{KEYWORDS}

Predator; trophic cascade; learning tool; programming; NetLogo

\section{Introduction}

Students find practical work relatively useful and enjoyable as compared with other science teaching and learning activities (Abrahams \& Millar, 2008). Cerini, Murray, and Reiss (2003) showed that $71 \%$ of surveyed students selected 'doing an experiment in class' as one of the three methods of teaching and learning science they found most enjoyable. Also; Roberts (2002) reported science, technology, engineering, and mathematics as key skills, and the students' learning experience could inspire and motivate themselves to study these subjects further at higher levels.

Individual based models (IBMs) are simulation models based on the interactions between individuals rather than on populations where population dynamics are usually a consequence of individual behavior. IBMs were developed from the need to understand and predict ecosystem complexity. Railsback $(2001,48)$ explained the IBM concept as 'build a model of an individual organism, build a model of the environment, and let a computer create multiple 
individual organisms and simulate the interactions of the individuals with each other and the environment' and stated that an IBM should address ecological concepts such as emergence, adaptation, fitness, state-based decisions, prediction, and computer implementation. Huhns and Singh (1998) defined the agents (individuals) as 'active and persistent (software) components that perceive, reason, act, and communicate'.

Although IBMs were born in the field of ecology, their development and use has grown steadily in recent years and the scope has been extended to several fields such as chemical engineering (Taherian and Mousavi, 2017), psychology (Schröder and Wolf, 2017) and health (Ufholz and Harlow, 2017). Within biology, IBMs have been applied to a large number of study areas including bird population dynamics (Parry et al., 2013), genetics (Pertoldi and Topping, 2004), land use effects on wildlife (Jepsen et al., 2005), microbiology (Oyebamiji et al., 2017), organic farming (Topping, 2011), plant evolution (Warren, Topping, and James, 2011) and statistics (Grazzini, Richiardi, and Tsionas, 2017).

Strong and comprehensive tools and platforms have also been developed in the context of ecological scientific research. For example, IBMs such as ALMaSS (Topping et al., 2003) and Weaver (Bilbao-Castro et al., 2015) are able to answer policy-related questions and facilitate management decision through the study of the effect of landscape structure, agricultural management and ecology on key animal species, and the evolution of their ecological interactions. Within the educational framework, computers and computer graphics must be integrated into traditional teaching (Bio, 2010), and although efforts have been made to introduce IBMs within the educational context (Betancourt and Más, 2012) the number of IBMs specifically oriented for teaching is scarce (Ginovart, 2014). In addition, integration of concepts across levels of complexity and the ability to synthesize and analyze information that connects conceptual domains are skills that students need to develop (Vision and Change, 2011). Accordingly, the objectives of this work were (1) to develop an IBM able to represent a three-level trophic cascade in the framework of biological pest control, (2) and to test its viability and acceptance in the real world using the model as a teaching tool with a group of high school students.

\section{Materials and methods}

\section{Programming platform}

EcoPred was developed in NetLogo (Wilensky, 1999). NetLogo is an open source multi-agent modeling environment that has been widely used in research and teaching contexts and is freely available from the NetLogo website. It is a complete environment for creating and running models that enables exploration of emergent phenomena and comes with an extensive library including models in several domains.

\section{Model description}

EcoPred simulates a top-down trophic cascade controlled by the pressure exerted by two spider species (models for a web-builder spider and a ground runner spider) on a fruit fly (model for the olive fruit fly Bactrocera oleae (Rossi, 1790)) in a simulated world that represents an olive orchard.

The model description follows the ODD (Overview, Design concepts, Details) protocol for describing individual- and agent-based models (Grimm et al. (2006), Grimm et al., 2010).

\section{Purpose}

EcoPred is a simple and easy to use IBM built on the NetLogo platform that aims to be an educational tool that can help teachers to explain four concepts related to ecology: (1) the concept of trophic cascade including three trophic levels (primary producers, herbivores and carnivores), (2) the concept 
of generalist and specialist species; (3) the concept of functional group (guild), and their practical application within a simulated arable land ecosystem, (4) the concept of biological pest control.

\section{Agents/entities, state variables, scales, and model parameters.}

The model uses three NetLogo types of entity: (1) turtles, (2) patches, and (3) an observer. Turtles correspond to the individuals, patches are spatial units composing the environment, and the observer is an entity that oversees everything that is going on in a two-dimensional world.

\subsection{Agents (entities).}

2.1.1. Observer: The observer is an entity that oversees everything that is going on in the world.

2.1.2. Patches: The world is two-dimensional and composed by a grid of patches. Each patch is a square piece of 'ground' over which turtles can move.

2.1.3. Turtles: Turtles are agents that move around and interact in the world.

2.1.3.1. Ground spiders (acting as natural enemies against flies). web-spiders).

2.1.3.2. Web-females (acting as natural enemies against flies and reproduction sites for

2.1.3.3. Web-males (acting as natural enemies against flies).

2.1.3.4. Flies (acting as pest).

2.1.3.5. Olives (representing olive trees that act as reproduction sites for flies).

2.1.3.6. Flowers (acting as food source for flies).

2.1.3.7. Stones (acting as reproduction sites for ground spiders)

\subsection{State variables:}

\subsubsection{Turtles}

2.2.1.1. Sex (constant variable): Web-spiders are divided in males (allowed to move) and females (static).

2.2.1.2. Size and color (constant variables): Each turtle group present a constant size (given in size units) and color:

2.2.1.2.1. Stones: 4 size units and color brown.

2.2.1.2.2. Olives: 1.5 size units and color violet.

2.2.1.2.3. Flowers: 3.5 size units and color yellow.

2.2.1.2.4. Flies: 2 size units and color white.

2.2.1.2.5. Web-females: 3 size units and color black.

2.2.1.2.6. Web-males: 2 size units and color black

2.2.1.2.7. Ground spiders: 3 size units and color brown.

2.2.1.3. Energy (this variable changes along time): flies and spiders (web-females, webmales and ground spiders) start with 50 energy units.

2.2.1.4. Heading (variable): it controls the direction the turtle is facing. Flies, web-females, web-males and ground spiders turn left and right while moving ahead according to a certain interval of degrees (see also section 7.1.2.).

2.2.1.4.1. Flies and web-males: the number (integer) of degrees to turn is randomly selected within the interval $[0,20)$ to the right and $[0,20)$ to the left.

2.2.1.4.2. Ground-spiders: the number (integer) of degrees to turn is randomly selected within the interval $[0,50)$ to the right and $[0,50)$ to the left.

2.2.1.5. Identity number (constant variable): a single identification number is assigned to each turtle.

2.2.1.6. Spatial location (this variable may change along time): the position of each type of turtle is described in section 7 (see below).

2.2.2. Patches.

2.2.2.1. Color (constant variable): Constant and green. 
2.2.2.2. Spatial location (constant variable): Patches are stationary and arranged in a grid.

2.3. Spatial scale:

2.3.1. Patch. The model landscape is comprised by a grid of $31 \times 67$ square patches (i.e. 2257 area units). Space is a dimensionless variable.

2.3.2. World topology. The world has neither horizontal nor vertical limits. The world is a torus which means it is not bounded, but wraps, i.e. flies and spiders that reach the left border disappear and reappear on the right edge.

2.4. Temporal scale:

2.4.1. Tick. Time passes in discrete steps called 'ticks'. Time is a dimensionless variable.

\subsection{Model parameters:}

2.5.1. Initial number of flowers: an integer within the interval $[0,100]$.

2.5.2. Initial number of stones: an integer within the interval $[0,50]$.

2.5.3. Initial number of olives: an integer within the interval $[0,100]$.

2.5.4. Initial number of flies: an integer within the interval $[0,50]$.

2.5.5. Initial number of web-females: an integer within the interval $[0,20]$.

2.5.6. Initial number of web-males: an integer within the interval $[0,20]$.

2.5.7. Initial number of ground-spiders: an integer within the interval $[0,20]$.

\section{Process overview and scheduling.}

3.1. Which agents do what processes? EcoPred incorporates two levels of processes, low level processes (i.e. carried out by turtles) and high-level processes (i.e. carried out by the Observer). Each process within each category is indicated and followed by the agent that performs it:

3.1.1. Low level processes: move (flies and web-males), move-b (ground-spiders), fed (flies), reproduce (flies), fed-a (web-females), reproduce-a (web-females), fed-b (web-males), reproduce-b (web-males), move-b (web-males), fed-c (ground-spiders), reproduce-c (ground-spiders),, death (flies, web-females, web-males and ground-spiders), and energy loss (flies, web-females, web-males and ground-spiders).

3.1.2. High-level processes: stop (if more than 2000 flies are counted within the crop, the system stops and show the message 'Your crop have been invaded!'; if more than 1000 web-females, web-males or ground-spiders are counted within the crop the system stops; if the number of flies reaches zero, the system stops), plot (the number of flies and spiders are plotted along time at each tick), count the number of turtles (the number of flies and spiders is counted at each time tick), create the initial population of turtles, and introduce some individuals of web-males and groundspiders (simulating immigration into the crop).

\subsection{Order of processes}

Firstly, the model checks the stop conditions described in section 3.1.2. and one web-male and one ground-spider are created. Then, flies move, feed, reproduce, die, and alive individuals lose energy, then web-females feed, reproduce, die, and lose energy, then web-males move, feed, reproduce, die, and alive individuals lose energy. Finally, ground-spiders move, feed, reproduce, some individuals enters the world, die and lose energy. The whole order and processes are represented at Figure 1.

3.3. When are state variables updated? During the simulation of processes or actions with the different entities, the turtles are called in a different random order in each time step (asynchronous updating). Each turtle completes the full set of actions requested before passing on control to the next agent at each time tick. 


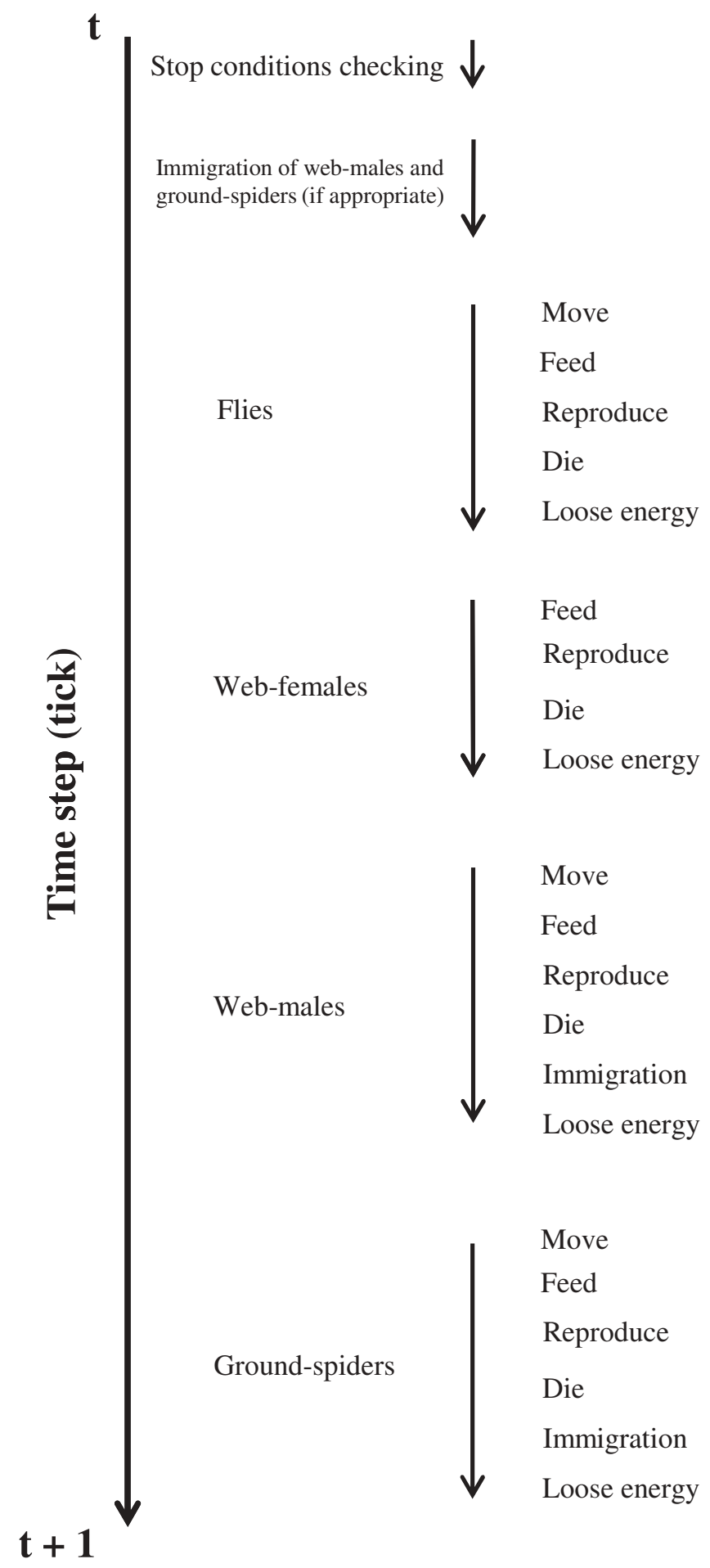

Figure 1. Schematic view of the trophic levels and processes included in EcoPred along a time step (tick). 


\section{Design concepts.}

4.1. Basic principles.: EcoPred is based on four general concepts related to ecology, (1) the trophic cascade, (2) generalist and specialist species, (3) functional traits, and (4) biological pest control.

4.1.1. The concept of three-level trophic cascade was introduced by Hairston, Smith, and Slobodkin (1960) in which three trophic levels are food-limited and present interspecific competition among levels that regulates the population of each level. Our model considers the primary producers (flowers), herbivores (flies) and carnivores (web and ground-spiders).

4.1.2. The concept of generalist or euryphagous species (i.e. a species that consumes a wide variety of prey species) and specialist or stenophagous species (i.e. a species that consume only particular parts of their prey, feed on only a narrow range of closely related species or even just a single species) (Begon, Townsend, and Harper, 2006). Our model considers both groups, the generalist predators are represented by the ground-spiders that feed on flies and web-males, and specialist predators represented by web-spiders that only feed on flies.

4.1.3. The concept of functional traits. Functional traits can be seen as key characteristics by which single species and groups of species influence ecosystem properties (de Bello et al., 2010) and are defined as a feature of an organism, which has demonstrable links to the organism's function (Lavorel et al., 1997). Consequently, 'functional groups' or guilds of species can be defined as an assemblage of organisms with similar functional trait attributes (Harrington et al., 2010), or in other words, species using the same class of resources in a behaviorally similar way (Simberloff and Dayan, 1991). Our model represents two guilds among predators, spiders inhabiting the ground, and webbuilder spiders. Each functional group behaves and feeds differently, ground spiders move freely and are generalist predators that need stones as shelter to reproduce (the model assumes that there are no sexual dimorphism), and web-building spiders are specialists. In addition, web-females are immobile and their reproduction depends on whether a male (that move freely) finds a female.

4.1.4. The concept of biological pest control. Natural ecosystems and their component species experience a rapid loss as habitat is destroyed for human use and invaded by species from other biogeographical areas (van Driesche et al., 2010). In the 1990s, the insect biological control against environmental pests was raised as an independent goal (van Driesche, 1994). Our model simulates the effect of two predators (web and ground spiders) that represent natural enemies on a fly species that represent the olive fruit fly Bactrocera oleae (Rossi) (Diptera: Tephritidae), the key pest of the olive tree (Boccaccio and Petacchi, 2009) in the Mediterranean basin within the simulated olive crop.

4.2. Emergence. The model represents two extreme situations. If the initial number of predators (i.e. spiders) is zero, the pest (i.e. flies) blooms and invades the crop. On the contrary, if the number of predators is set at maximum, the population plot at the end of the simulation represent the biological control of flies (i.e. their population reaches zero) exerted by the specialist predator (i.e. web-spiders) and the generalist predator (i.e. ground spiders) being the former dominated by the latter.

4.3. Sensing. The mechanisms by which flies and spiders obtain information about food sources and reproduction processes are modeled explicitly.

4.3.1. Flies only feed if the agent they found is a flower.

4.3.2. Flies only reproduce if the agent they found is an olive.

4.3.3. Web-spiders only feed if the agent they found is a fly.

4.3.4. Web-spiders only reproduce if the agent they found is a web-spider.

4.3.5.Web-females only reproduce if the agent they found is a web-male. 
4.3.6. Ground-spiders only feed if the agent they found is a fly or a web-male spider.

4.3.7. Ground-spiders only reproduce if the agent they found is a stone.

4.4. Interaction. EcoPred incorporates both direct and indirect interactions; however they not involve communication between turtles.

4.4.1. Direct interactions:

4.4.1.1. Feeding of flies on flowers.

4.4.1.2. Predation of web-spiders over flies.

4.4.1.3. Predation of ground-spiders over flies.

4.4.1.4. Predation of ground-spiders over web-males.

4.4.1.5. Reproduction of flies (the fly only reproduce if an olive is found).

4.4.1.6. Reproduction of web-spiders (the spider only reproduces if a female or male is found respectively). found).

4.4.1.7. Reproduction of ground-spiders (the spider only reproduces if a stone is

4.4.2. Indirect interactions:

4.4.2.1. Competition for food (i.e. flies) among spiders. Competition for food among flies is not considered since during the time window they need to reach a pest outbreak the resources provided by flowers are assumed to be unlimited.

4.5. Stochasticity. Stochasticity is used in three processes related to the position of the turtles: (1) to place the turtles over the world at the initial time tick, (2) to place the hatched flies and spiders, and (3) to define the heading pattern of moving individuals. Stochasticity is not used to cause model events or behaviors to occur.

4.6. Collectives. The individuals do not form aggregations in EcoPred.

4.7. Observation. The 'View' window within the user interface is a visual representation of the world of turtles and patches where the user can see the evolution of the system at real time. The number of flies, web-spiders (i.e. web-females and web-males) and ground-spiders is collected and plotted at each time tick by the observer to be interpreted. Also, the whole dataset (i.e. the results at each time tick) can be manually exported as an excel file (.csv extension) for further analyses if desired.

5. Initialization. The aim of the model is to analyze the consequences of its initial state, i.e., how the population of flies and spiders change along time as a function of the initial number of each turtle. For this purpose, the number of individuals can be arbitrarily selected in the GUI using sliders within the following ranges:

Flowers: $0-100$ individuals.

Stones: $0-50$ individuals.

Olives: $0-100$ individuals.

Flies: $0-50$ individuals.

Web-females: 0-20 individuals.

Web-males: 0-20 individuals.

Ground-spiders: 0-20 individuals.

6. Input data. The model does not use input from external sources such as data files or other models to represent processes that change over time.

7. Submodels.

7.1. Low level processes:

7.1.1. Loose energy (flies, web-females, web-males, ground-spiders)

$$
E_{(t+1)}=E_{(t)}-1
$$

where $\mathrm{E}$ denotes the amount of energy units the turtle owns, and $\mathrm{t}$ denotes the time tick.

7.1.2. Move (flies, web-males and ground-spiders): 
Flies, web-males and ground-spiders move endlessly around the world by advancing forward one unit of distance at each time step (note that since patches are squares, a step forward could occur within the same patch). The degree of turn to each side (heading) is defined by a random integer at each time step (see section 2.5.). Movement is independent of the density of turtles and patches can house more than one turtle.

7.1.3. Fed (flies):

$$
E_{(t+1)}=E_{(t)}+N_{(t)} \times 30
$$

where $\mathrm{E}$ denotes the number of energy units the fly has, $\mathrm{N}$ is the number of flowers on the patch, and $t$ the time tick. The rule applies if the fly finds a flower at a distance of one unit along its current heading at $\mathrm{t}+1$.

7.1.4. Reproduce (flies):

$$
N_{(t+1)}=4 \times N_{0(t)}
$$

where $\mathrm{N}_{0}$ denotes the number of ascendants in a patch, $\mathrm{N}$ is the number of ascendants plus descendants ( 3 flies will hatch), and t the time tick. The rule applies if a fly enters a patch occupied by an olive, then the flies hatched are randomly positioned within the world. The energy is equally divided between the hatched flies and the parent.

7.1.5. Fed-a (web-females):

$$
E_{W F(t+1)}=E_{W F(t)}+N_{(t)} \times 5
$$

where $\mathrm{E}_{\mathrm{WF}}$ denotes the number of energy units the web-female has, $\mathrm{N}$ is the number of flies on the patch, and $t$ the time tick. The rule applies if the web-female spider finds a fly at a distance of one unit along its current heading at $t+1$. Once the spider feeds, the flies found die.

7.1.6. Reproduce-a (web-females):

$$
N_{W F(t+1)}=2 \times N_{W F 0(t)}
$$

where $\mathrm{N}_{\mathrm{WF}}$ denotes the number of ascendants at the patch, $\mathrm{N}_{\mathrm{WF}}$ is the number of ascendants plus descendants ( 1 web-female will hatch), and $t$ is the time tick. The rule applies if a web-male spider enters a patch occupied by a web-female, then the web-females hatched are randomly positioned within the world. The energy is equally divided between the hatched spiders and the parent.

7.1.7. Fed-b (web-males):

$$
E_{W M(t+1)}=E_{W M(t)}+N_{(t)} \times 5
$$

where $\mathrm{E}_{\mathrm{WM}}$ denotes the number of energy units the web-male has, $\mathrm{N}$ is the number of flies on the patch, and the time tick. The rule applies the web-male spider finds one fly at a distance of one unit along the turtle current heading at $t+1$. Once the spider feeds, the flies found die.

7.1.8. Reproduce-b (web-males):

$$
N_{W M(t+1)}=2 \times N_{W M 0(t)}
$$

where $\mathrm{N}_{\mathrm{WM} 0}$ denotes the number of ascendants at the patch, $\mathrm{N}_{\mathrm{WM}}$ is the number of ascendants plus descendants ( 1 web-male will hatch), and $t$ is the time tick. The rule applies if the patch the web-male enters is occupied by a web-female spider, and then the web-males hatched are randomly positioned within the world. The energy is equally divided between the hatched spiders and the parent.

7.1.9. Fed-c (ground-spiders):

$$
E_{G S(t+1)}=E_{G S(t)}+N_{F(t)} \times 10+N_{W M(t)} \times 10
$$


where $\mathrm{E}_{\mathrm{GS}}$ denotes the number of energy units the ground-spider has, $\mathrm{N}_{\mathrm{F}}$ is the number of flies on the patch, $\mathrm{N}_{\mathrm{WM}}$ is the number of web-male spiders on the patch, and the time tick. The rule applies if the ground-spider finds a fly at a distance of one unit along the turtle current heading, or a web-male spider in the same patch the ground-spider entered at $t+1$. Once the spider feeds, the flies/web-males found die.

7.1.10. Reproduce-c (ground-spiders):

$$
N_{G S(t+1)}=2 \times N_{G S O(t)}
$$

where $\mathrm{N}_{\mathrm{GS} 0}$ denotes the number of ascendants in a patch where a stone is present, $\mathrm{N}_{\mathrm{GS}}$ is the number of ascendants plus descendants ( 1 ground-spider will hatch), and $t$ the time tick. The ground-spiders hatched are randomly positioned within the world. The energy is equally divided between the hatched flies and the parent.

7.1.11. Death (flies, web-females, web-males, and ground-spiders).

If the condition $\mathrm{E}_{(\mathrm{t}+1)}=0$, where $\mathrm{E}$ denotes the number of energy units is met, the turtle dies. 7.2.High-level processes:

7.2.1. Stop (flies, web-females, web-males, and ground-spiders):

If the condition $\mathrm{N}_{\mathrm{F}(\mathrm{t})}=0$ is met, where $\mathrm{N}_{\mathrm{F}}$ denotes the total number of flies and $\mathrm{t}$ is the time tick, the system stops.

If the condition $\mathrm{N}_{\mathrm{F}(\mathrm{t})}>2000$ is met, where $\mathrm{N}_{\mathrm{F}}$ denotes the total number of web-females and $\mathrm{t}$ is the time tick, the system stops.

If the condition $\mathrm{N}_{\mathrm{WF}(\mathrm{t})}>1000$ is met, where $\mathrm{N}_{\mathrm{WF}}$ denotes the total number of web-females and $\mathrm{t}$ is the time tick, the system stops.

If the condition $\mathrm{N}_{\mathrm{WM}(\mathrm{t})}>1000$ is met, where $\mathrm{N}_{\mathrm{WM}}$ denotes the total number of web-males and $\mathrm{t}$ is the time tick, the system stops.

If the condition $\mathrm{N}_{\mathrm{GS}(\mathrm{t})}>1000$ is met, where $\mathrm{N}_{\mathrm{GS}}$ denotes the total number of ground-spiders and $\mathrm{t}$ is the time tick, the system stops.

7.2.2. Plot. The number of flies and spiders are plotted along time at each tick as:

$$
\begin{gathered}
\text { Flies }=\mathrm{N}_{\mathrm{F}(\mathrm{t})} \\
\text { Web-spiders }=\mathrm{N}_{\mathrm{WF}(\mathrm{t})}+\mathrm{N}_{\mathrm{WM}(\mathrm{t})} \\
\text { Ground-spiders }=\mathrm{N}_{\mathrm{GS}(\mathrm{t})}
\end{gathered}
$$

where $t$ denotes the time tick, $\mathrm{N}_{\mathrm{F}(\mathrm{t})}$ is the number of flies at time $\mathrm{t}, \mathrm{N}_{\mathrm{WF}(\mathrm{t})}$ is the number of webfemale spiders at time $t, \mathrm{~N}_{\mathrm{WM}(\mathrm{t})}$ is the number of web-male spiders at time $t$, and $\mathrm{N}_{\mathrm{GS}(\mathrm{t})}$ is the number of ground-spiders at time $\mathrm{t}$.

\section{Model performance}

Twenty simulation runs were run and plotted together to test the model performance at each extreme situation, i.e., the evolution of the fly population along time in the absence of predators starting with the maximum number of flies, and the evolution of the fly population in the presence of the maximum number of predators. In order to assess the pattern stability, for each situation and key turtle (i.e. flies, web-spiders and ground-spiders) the mean and the standard error of the number of individuals were calculated at each time step (tick) and plotted together to allow visual comparison with the raw simulations. Finally, the mean of the number of individuals among simulations at each time step was modeled according to the observed pattern. Calculation of estimates and data fitting were performed in (R Core Team, 2017).

\section{Method testing at classroom}

In order to assess the acceptance, performance and interest of students on the use of IBMs and more specifically of our model, we used EcoPred with 26 students of 16 years old coming from three 
different secondary schools of Bragança (Portugal). The students were allowed to explore and use, for $1 \mathrm{~h}$, a simplified version of our model during an activity called 'EcoPred: from the field to the computer, a virtual ecosystem' within the 'Science and Technology Week 2017. The activity was divided into three blocks of 20 minutes; during the first block, three aspects were explained: (1) the concept of IBMs, (2) the concept of biological pest control, and (3) how EcoPred can be used to reproduce the effect of a predator on a pest. During the second block, the students were allowed to run as many simulations as they wanted changing the initial parameters in two ways, (1) setting the initial number of predators at zero and the number of flies at maximum, and (2) setting the initial number of flies and predators at maximum. Then, the simulation results with and without predators were briefly discussed. Finally, during the third block, the students were encouraged to modify the code (accompanied by the monitor) in order to change the identity of the turtles (shape, size and color). The activity took place at the School of Agriculture (Polytechnic Institute of Bragança) and a survey was carried out at the end of the activity by asking to the students eight yes/no key questions:

1. Have you ever used any kind of simulation model?

2. Did this activity seem fun to you?

3. Did this activity seem complicated?

4. Would you like to continue creating your own ecosystem?

5. Did you have previous programming knowledge?

6. Would you like to learn how to program?

7. In your opinion, is a simulation model useful for learning?

8. Do you think that programming is useful in real life?

\section{Results}

\section{Interface, simulations, and model performance}

The designed graphical user interface encompasses a time speed control device, a set of devices used to select the initial number of each agent (turtle), a live data plot used to register the fly and spider's population changes along time, a counter for the number of flies, web-spiders and ground-spiders, and the 2D-world that represent the agents and interactions (Figure 2). In general, the pattern on the evolution of each species' population was consistent among simulation runs (Figure 3(a,b) and supported by the amount of standard deviation (Figure 3(c,d). In terms of modeling, the fly population in the absence of predators followed an exponential growth until the stop condition was reached (i.e. > 2000 flies) given by:

$$
y \sim \exp (a+b \times x)
$$

where $y$ denotes the number of flies and $x$ is the time step (tick), $\mathrm{a}=4.31 \pm 0.069$ (estimate $\pm \mathrm{SE}$ ) $(\mathrm{t}=62.88 ; \mathrm{P}<0.001), \mathrm{b}=0.071 \pm 0.002$ (estimate $\pm \mathrm{SE})(\mathrm{t}=43.44 ; \mathrm{P}<0.001)$ (Figure 4(a)).

The evolution of the fly population in the presence of predators was fitted using local polynomial regression fitting (loess) with a degree of smoothing of $\alpha=0.5$, and polynomials of degree $=1$ that gave an equivalent number of parameters $=4.1$, and a residual standard error $=12.60$ (Figure $4(\mathrm{~b})$ ).

The evolution of the web-spiders' population was fitted using local polynomial regression fitting (loess) with a degree of smoothing of $\alpha=0.5$, and polynomials of degree $=1$ that gave an equivalent number of parameters $=4.1$, and a residual standard error $=6.82$ (Figure $4(\mathrm{~b})$ ).

The evolution of the ground-spiders' population was fitted using local polynomial regression fitting (loess) with a degree of smoothing of $\alpha=0.5$, and polynomials of degree $=1$ that gave an equivalent number of parameters $=4.1$, and a residual standard error $=7.05$ (Figure $4(\mathrm{~b})$ ). 


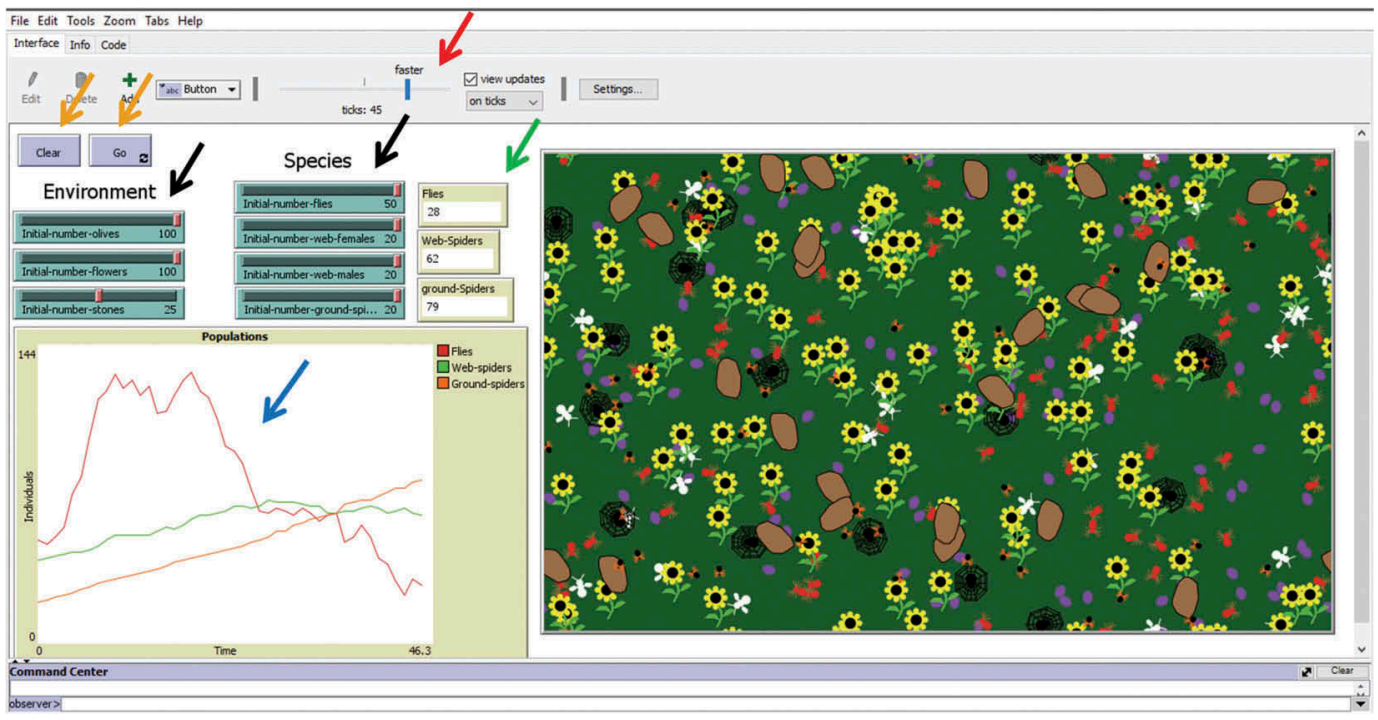

Figure 2. Aspect of the graphical user interface (GUI) of EcoPred. The red arrow indicates the time speed control device. The black arrows indicate the devices used to select the initial number of each agent (turtle). The blue arrow indicates the live data plot used to register the fly and spider's population changes along time. The green arrow indicates the individual counters for flies and spiders. The orange arrows indicate the buttons used to reset the initial conditions ('Clear') and initiate ('Go') a simulation.

\section{Method testing at classroom}

About two thirds of the students had never used a simulation model before, however the same amount of them had some previous programming knowledge (Table 1). Most of the students found the activity fun, not difficult, and would like to continue developing the ecosystem (Table 1). Also, the vast majority of students said that they would like to learn programming, found the simulation model useful for learning, and thought that programming could be useful in real life (Table 1).

\section{Discussion}

Our model represents consistently the relationship between the populations of the trophic levels of a three-level trophic cascade along time. The concepts related to population dynamics are mandatory knowledge for environmental science students, especially those related to areas such as agronomy, ecology, microbiology and zoology. EcoPred proved to be useful and interesting to students during an activity oriented to discuss the role of different types of predators on the ability to control a pest within a simulated agroecosystem. Two extreme possible situations in the context of biological pest control were assessed during the activity using EcoPred, i.e. how the fly population evolves with and without predators during the simulation. Our approach allows a discussion of the implications of an up-to-date strategy of pest control in agricultural management such as the biological pest control at each situation in terms of relevant ecology-related concepts including trophic cascade, generalist and specialist species and species' guilds.

Beyond the basic approach we followed, some more questions can be posed by teachers such as:

- How would predator populations evolve if the system stop conditions were eliminated?

- How would the fly population evolve if there were just the specialist predator present? 

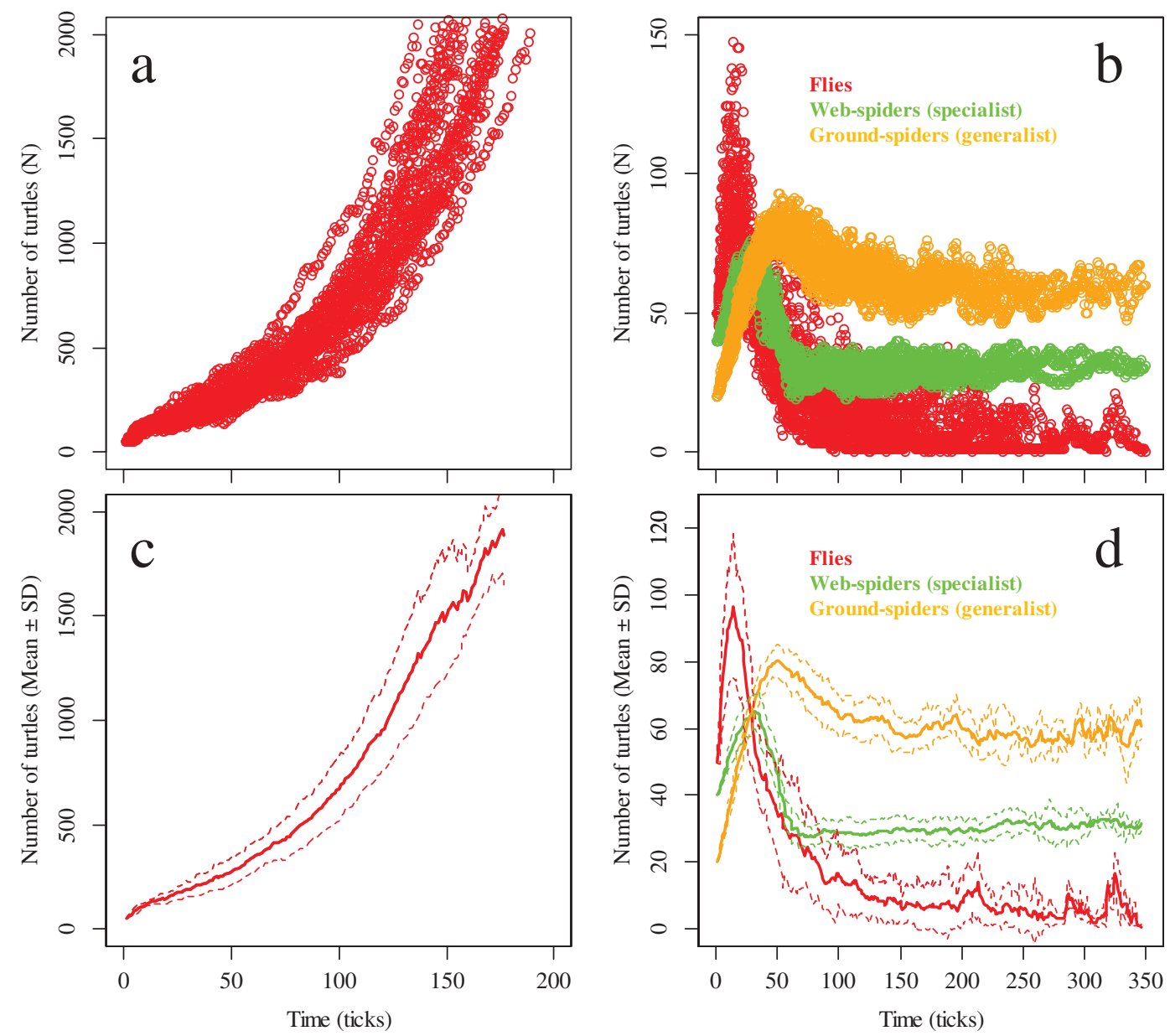

Figure 3. Results of 20 simulation runs for each of two extreme situations (flies with and without predators) in EcoPred. (a) evolution of the fly population at each time step (tick) (raw data) without predators; (b) evolution of the fly and predators' population together at each time step (tick) (raw data); (c) pattern followed by the mean number of flies along time (ticks) without predators, and (d) pattern followed by the mean number of flies, web-spiders and ground-spiders along time (ticks). In all cases the initial number of individuals was established at maximum. In a and b, circles represent the abundance of turtles at each time step. In c and d, continuous lines represent the evolution of the average abundance of turtles, and dashed lines represent the evolution of its standard deviation along time.

- How would the fly population evolve if there were just the generalist predator present?

- How would the population of flies and spiders evolve if the initial amount of resources changes?

In addition, our model parameterization in terms of initial conditions, such as the initial amount of energy, the maximum number of turtles, the amount of patches moved in each tick or the behavior of spiders was established ad hoc; however, the flexibility of NetLogo enables these to be easily changed and to include more agents into the trophic network such as parasitoids, more predator species and different food and reproductive resources for each trophic level. For example, different population dynamic models such as the Lotka-Volterra equations for predator-prey systems (Yorke and Anderson, 1973) could be implemented within the trophic cascade if desired towards a more realistic model development. Also, following the open source paradigm, students of higher levels (e.g. university level) or students specifically oriented to programming courses can be encouraged to statistically analyze the 

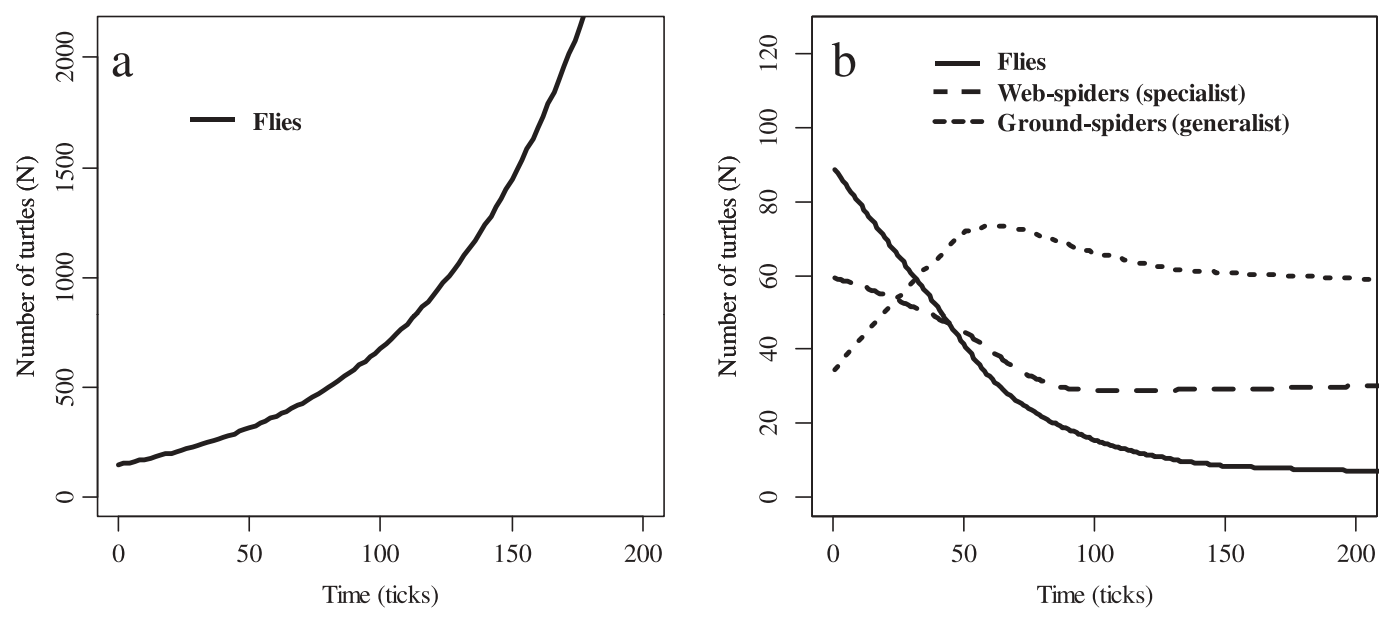

Figure 4. Fitted models for two extreme situations (flies with and without predators) simulated in EcoPred. (a) pattern of the pest (flies) population evolution without the pressure exerted by predators, and (b) pattern of the pest (flies), specialist predator (web-spiders) and generalist predator (ground-spiders) populations running the three-level trophic cascade with the initial number of individuals established at maximum. Dots were omitted within the charts to increase clarity.

Table 1. Results of the obtained with 26 students from high schools who tested the EcoPred model during their participation in the 'science and technology week 2017' in the school of agriculture, Bragança, Portugal. N: number of students surveyed; DK/NA: Don't know/No answer.

\begin{tabular}{llcrc}
\hline Question & N & Yes (\%) & No (\%) & DK/NA (\%) \\
\hline 1 & 26 & 30.77 & 61.54 & 7.69 \\
2 & 26 & 96.15 & 3.85 & 0.00 \\
3 & 26 & 19.23 & 80.77 & 0.00 \\
4 & 26 & 88.46 & 11.54 & 0.00 \\
5 & 26 & 69.23 & 30.77 & 0.00 \\
6 & 26 & 96.15 & 3.85 & 0.00 \\
7 & 26 & 92.31 & 7.69 & 0.00 \\
8 & 26 & 96.15 & 3.85 & 0.00 \\
\hline
\end{tabular}

outputs of EcoPred in R software; for example, modeling the different responses as a function of time, or finding the optimal model among competing models for a species' population response using specific criteria such as goodness of fit $\left(\mathrm{R}^{2}\right)$ or the Akaike information criterion (AIC) (Aho, Derryberry, and Peterson, 2014).

Simulation is a method increasingly used for both research and teaching. Therefore, manipulating a virtual ecosystem through a simple programming language can be a beneficial approach for students. In fact, programming and data analysis are increasingly demanded skills in a world more and more dominated by open source software. Our survey suggested that students are interested in gaining programming skills, they recognized simulation-based approaches as a useful and enjoyable tool and they would like to continue learning in this context. EcoPred integrates both components at the same time, (1) ecological learning within a biological control framework and (2) programming learning, with the advantage that each user can expand the complexity of his model in an unlimited way using user-friendly code syntax on a free platform.

\section{Acknowledgments}

This work was funded by the Portuguese Foundation for Science and Technology (FCT) through the project PTDC/AGR-PRO/111123/2009 and ReNATURE - Valorization of the Natural Endogenous Resources of the Centro 
Region" funded by Centro 2020 (Centro-01-0145-FEDER-000007). This manuscript is part of Jacinto Benhadi-Mar ín's Ph.D. thesis. Jacinto Benhadi-Marín is grateful to the Portuguese Foundation for Science and Technology for financial support through the Ph.D. grant SFRH/BD/97248/2013.

\section{Data accessibility}

EcoPred (application and code) is available from https://github.com/jbenma/EcoPred. The NetLogo software and user manual are available from https://ccl.northwestern.edu/netlogo/

\section{Disclosure statement}

No potential conflict of interest was reported by the authors.

\section{Funding}

This work was supported by the Portuguese Foundation of Science and Technology [PTDC/AGR-PRO/111123/200, SFRH/BD/97248/2013]; Centro 2020 [Centro-01-0145-FEDER-000007].

\section{ORCID}

Jacinto Benhadi-Marín (iD http://orcid.org/0000-0002-9804-4145

José Alberto Pereira (iD) http://orcid.org/0000-0002-2260-0600

José Paulo Sousa (iD http://orcid.org/0000-0001-8045-4296

Sónia A.P. Santos (iD) http://orcid.org/0000-0003-1500-6360

\section{References}

Abrahams, I., and R. Millar. 2008. "Does Practical Work Really Work? A Study of the Effectiveness of Practical Work as A Teaching and Learning Method in School Science." International Journal of Science Education 30 (14): 1945-1969. doi:10.1080/09500690701749305.

Aho, K., D. Derryberry, and T. Peterson. 2014. "Model Selection for Ecologists: The Worldviews of AIC and BIC." Ecology 95: 631-636. doi:10.1890/13-1452.1.

Begon, M., C. R. Townsend, and J. L. Harper. 2006. ECOLOGY. From Individuals to Ecosystems. Malden: WileyBlackwell.

Betancourt, J. A., and M. H. Más. 2012. Agent Based Models for Public Health Teaching. Memorias Convención Internacional de Salud Pública. Cuba Salud 2012. La Habana 3-7 de diciembre de 2012. Ministerio de Salud Pública de Cuba, La Habana: Cuba. ISBN 978-959-212-811-8.

Bilbao-Castro, J. R., G. Barrionuevo, D. Ruiz-Lupión, L. G. Casado, and J. Moya-Laraño. 2015. "Weaver, A Multiagent, Spatial-Explicit and High-Performance Framework to Study Complex Ecological Networks." In: Bajo J. et al. (eds.), Highlights of Practical Applications of Agents, Multi-Agent Systems, and Sustainability The PAAMS Collection. PAAMS 2015. Communications in Computer and Information Science, 524. Cham, Switzerland: Springer.

Bio. 2010. Transforming Undergraduate Education for Future Research Biologists (2003) Committee on Undergraduate Biology Education to Prepare Research Scientists for the 21st Century, National Research Council. Washington, DC: National Academies Press. Accessed 21 September 2017. http://www.nap.edu/open book.php?isbn=0309085357

Boccaccio, L., and R. Petacchi. 2009. "Landscape Effects on the Complex of Bactrocera Oleae Parasitoids and Implications for Conservation Biological Control.” BioControl 54: 607-616. doi:10.1007/s10526-009-9214-0.

Cerini, B., I. Murray, and M. Reiss. 2003. Student Review of the Science Curriculum. Major Findings. London: Planet Science/Institute of Education University of London/Science Museum. Accessed 21 September 2017. http://www. academia.edu/494193/The_student_review_of_the_science_curriculum.

de Bello, F., S. Lavorel, S. Díaz, R. Harrington, J. H. C. Cornelissen, R. D. Bardgett, M. P. Berg, et al. 2010. “Towards an Assessment of Multiple Ecosystem Processes and Services via Functional Traits." Biodiversity Conservation 19: 2873-2893. doi:10.1007/s10531-010-9850-9.

Ginovart, M. 2014. "Discovering the Power of Individual-Based Modelling in Teaching and Learning: The Study of a Predator-Prey System.” Journal of Science Education and Technology 23: 496-513. doi:10.1007/s10956-0139480-6. 
Grazzini, J., M. G. Richiardi, and M. Tsionas. 2017. “Bayesian Estimation of Agent-Based Models.” Journal of Economic Dynamics and Control 77: 26-47. doi:10.1016/j.jedc.2017.01.014.

Grimm, V., U. Berger, F. Bastiansen, S. Eliassen, V. Ginot, J. Giske, J. Goss-Custard, et al. 2006. "A Standard Protocol for Describing Individual-Based and Agent-Based Models.” Ecological Modelling 198: 115-126. doi:10.1016/j.ecolmodel.2006.04.023.

Grimm, V., U. Berger, D. L. de Angelis, J. G. Polhill, J. Giske, and S. F. Railsback. 2010. “The ODD Protocol: A Review and First Update.” Ecological Modelling 221: 2760-2768. doi:10.1016/j.ecolmodel.2010.08.019.

Hairston, N. G., F. E. Smith, and L. B. Slobodkin. 1960. "Community Structure, Population Control and Competition." American Naturalist 94: 421-425. doi:10.1086/282146.

Harrington, R., C. Anton, T. P. Dawson, F. de Bello, C. K. Feld, J. R. Haslett, T. Kluvánkova-Oravská, et al. 2010. "Ecosystem Services and Biodiversity Conservation: Concepts and a Glossary." Biodiversity Conservation 19: 2773-2790. doi:10.1007/s10531-010-9834-9.

Huhns, M. N., and M. P. Singh, Eds. 1998. Readings in Agents. San Francisco, CA: Morgan Kaufmann.

Jepsen, J. U., C. J. Topping, P. Odderskær, and P. N. Andersen. 2005. "Evaluating Consequences of Land-Use Strategies on Wildlife Populations Using Multiple-Species Predictive scenarios.Agriculture." Ecosystems and Environment 105 (4): 581-594. doi:10.1016/j.agee.2004.08.007.

Lavorel, S., S. McIntyre, J. Landsber, and T. D. A. Forbes. 1997. "Plant Functional Classification: From General Groups to Specific Groups Based on Response to Disturbance.” Trends in Ecology \& Evolution 12: 474-478. doi:10.1016/S0169-5347(97)01219-6.

Oyebamiji, O. K., D. J. Wilkinson, P. G. Jayathilake, T. P. Curtis, S. P. Rushton, B. Li, and P. Gupta. 2017. "Gaussian Process Emulation of an Individual-Based Model Simulation of Microbial Communities." Journal of Computational Science 22: 69-84. doi:10.1016/j.jocs.2017.08.006.

Parry, H. R., C. J. Topping, M. C. Kennedy, N. Boatman, and A. Murray. 2013. “The Use of Agent-Based Models for Studying the Response of Bird Populations to Landscape Change." Environmental Software and Modelling 45: 104-115. doi:10.1016/j.envsoft.2012.08.006.

Pertoldi, C., and C. J. Topping. 2004. "Impact Assessment Predicted by Means of Genetic Agent-Based Modeling." Critical Reviews in Toxicology 34 (6): 487-498. doi:10.1080/10408440490519795.

R Core Team. 2017. R, a Language and Environment for Statistical Computing. Vienna, Austria: R Foundation for Statistical Computing. Accessed 21 September 2017. http://www.R-project.org

Railsback, S. F. 2001. "Concepts from Complex Adaptive Systems as a Framework for Individual-Based Modelling." Ecological Modelling 139: 47-62. doi:10.1016/S0304-3800(01)00228-9.

Roberts, S. G. 2002. SET for Success. The Supply of People with Science, Technology, Engineering and Mathematics Skills. London: HM Treasury. Accessed 21 September 2017. http://dera.ioe.ac.uk/4511/.

Schröder, T., and I. Wolf. 2017. "Modeling Multi-Level Mechanisms of Environmental Attitudes and Behaviours, the Example of Car Sharing in Berlin." Journal of Environmental Psychology 52: 136-148. doi:10.1016/j. jenvp.2016.03.007.

Simberloff, D., and T. Dayan. 1991. "The Guild Concept and the Structure of Ecological Communities." Annual Review of Ecology, Evolution and Systematics 22: 115-143. doi:10.1146/annurev.es.22.110191.000555.

Taherian, M., and S. M. Mousavi. 2017. "Modeling and Simulation of Forward Osmosis Process Using Agent-Based Model System.” Computers and Chemical Engineering 100: 104-118. doi:10.1016/j.compchemeng.2017.02.005.

Topping, C. J. 2011. "Evaluation of Wildlife Management through Organic Farming." Ecological Engineering 37: 2009-2017. doi:10.1016/j.ecoleng.2011.08.010.

Topping, C. J., T. S. Hansen, T. S. Jensen, J. U. Jepsen, F. Nikolajsen, and P. Odderskær. 2003. “ALMaSS, an Agent-Based Model for Animals in Temperate European Landscapes.” Ecological Modelling 167 (1-2): 65-82. doi:10.1016/S0304-3800(03)00173-X.

Ufholz, K. E., and L. L. Harlow. 2017. "Modeling Multiple Health Behaviors and General Health." Preventive Medicine 105: 127-134. doi:10.1016/j.ypmed.2017.08.003.

van Driesche, R. G. 1994. “Classical Biological Control of Environmental Pests.” Florida Entomologist 77: $20-33$. doi: $10.2307 / 3495870$.

van Driesche, R. G., R. I. Carruthers, T. Center, M. S. Hoddle, J. Hough-Goldstein, L. Morin, L. Smith, et al. 2010. "Classical Biological Control for the Protection of Natural Ecosystems." Biological Control 54 (Supplement 1): S2-S33. doi:10.1016/j.biocontrol.2010.03.003.

Vision and Change. 2011. "Vision and Change in Undergraduate Biology Education. A Call to Action." In Final Report of a National Conference Organized by the American Association for the Advancement of Science with Support from the National Science Foundation, edited by C. Brewer and D. Smith, Washington, DC: American Association for the Advancement of Science. Accessed 9 September 2018. http://visionandchange.org/files/2011/ 03/Revised-Vision-and-Change-Final-Report.pdf 
Warren, J., C. J. Topping, and P. James. 2011. “An Evolutionary Modelling Approach to Understanding Factors behind Plant Invasiveness and Community Susceptibility to Invasion.” Journal of Evolutionary Ecology 24: 2099-2109.

Wilensky, U. 1999. NetLogo. Center for Connected Learning and Computer-Based Modeling. Evanston, IL: Northwestern University. Accessed 9 September 2018. https://ccl.northwestern.edu/netlogo/.

Yorke, J. A., and W. N. Anderson Jr. 1973. "Predator-Prey Patterns (Volterra-Lotka Equations)." Pnas 70 (7): 2069-2071. doi:10.1073/pnas.70.7.2069. 